OPEN ACCESS

Edited by:

Babak Behnam,

National Sanitation Foundation

International, United States

Reviewed by:

Anthony Pak-Yin Liu,

The University of Hong Kong, Hong

Kong SAR, China

Hossein Khiabanian,

Rutgers, The State University of New

Jersey, United States

*Correspondence:

Deze Jia

dezejia_q/@126.com

${ }^{\dagger}$ These authors share first authorship

Specialty section:

This article was submitted to Genetics of Common and Rare

Diseases,

a section of the journal

Frontiers in Genetics

Received: 13 June 2021 Accepted: 24 January 2022

Published: 23 February 2022

Citation:

Li X, Xue H, Luo N, Han T, Li M and Jia D (2022) The First Case Report of a

Patient With Oligodendroglioma Harboring CHEK2 Germline Mutation.

Front. Genet. 13:718689.

doi: $10.3389 /$ fgene.2022.718689

\section{The First Case Report of a Patient With Oligodendroglioma Harboring CHEK2 Germline Mutation}

\author{
Xueen $\mathrm{Li}^{1 \dagger}$, Hao Xue ${ }^{1}$, Ningning $\mathrm{Luo}^{2}$, Tiantian $\mathrm{Han}^{2}$, Mengmeng $\mathrm{Li}^{2}$ and Deze Jia ${ }^{1 *}$ \\ ${ }^{1}$ Department of Neurosurgery, Qilu Hospital of Shandong University and Brain Science Research Institute, Shandong \\ University, Jinan, China, ${ }^{2}$ The Medical Department, Jiangsu Simcere Diagnostics Co., Ltd, Nanjing Simcere Medical \\ Laboratory Science Co., Ltd, The State Key Lab of Translational Medicine and Innovative Drug Development, Jiangsu \\ Simcere Diagnostics Co., Ltd, Nanjing, China
}

Introduction: CHEK2 (Checkpoint kinase 2) germline mutations were associated with an elevated risk of breast cancer, colorectal cancer, and other familiar cancers. Lossof-function variants in CHEK2 are known to be pathogenic. Germline CHEK2 mutations have also been observed in medulloblastoma and primary glioblastomas. Currently, there is no direct evidence supporting the relationship of CHEK2 with central nervous system tumors.

Case presentation: A case of an oligodendroglioma patient harboring the germline CHEK2 p.R137* mutation was reported. CHEK2 p.R137* mutation occurred in the forkhead-associated domain. Given the absence of other known genetic predisposing risk factors, we considered that oligodendroglioma might be associated with the CHEK2 mutation. The patient in our case might have a high risk of breast cancer and other multiple primary tumors. Her siblings and offspring would have a 50\% chance of having the same variant.

Conclusion: We reported a case of an oligodendroglioma patient with a family history of gastrointestinal tumors harboring the germline CHEK2 pathogenic variation. This is the first report of the association between the CHEK2 pathogenic variation and brain tumors that warrants further validation in larger cohorts.

Keywords: oligodendroglioma, germline mutation, CHEK2 gene, potential genetic predisposition, CNS tumor

\section{INTRODUCTION}

Checkpoint kinase 2 (CHEK2), a cell cycle checkpoint regulator gene and a tumor suppressor gene, is involved in DNA repair, cell cycle arrest, and apoptosis through the role in the homologous recombination DNA repair pathways (Stolarova et al., 2020). CHEK2 is activated by an ataxia telangiectasia mutated (ATM) kinase and is in turn capable of phosphorylating several substrates including p53 and BRCA1, leading to cell cycle arrest, apoptosis, and DNA repair (Bartek and Lukas, 2003). Heterozygous loss-of-function germline pathogenic variants in CHEK2 are associated with an increased risk for breast cancer with a lifetime risk of 25-39\% (Jalilvand et al., 2017).

CHEK2 1100delC allele is the most widely employed mutation point and is associated with LiFraumeni syndrome. Missense mutation I157T, another common CHEK2 pathogenic variant, is reported to be associated with a higher risk for colon cancer (Jalilvand et al., 2017). The effects of 

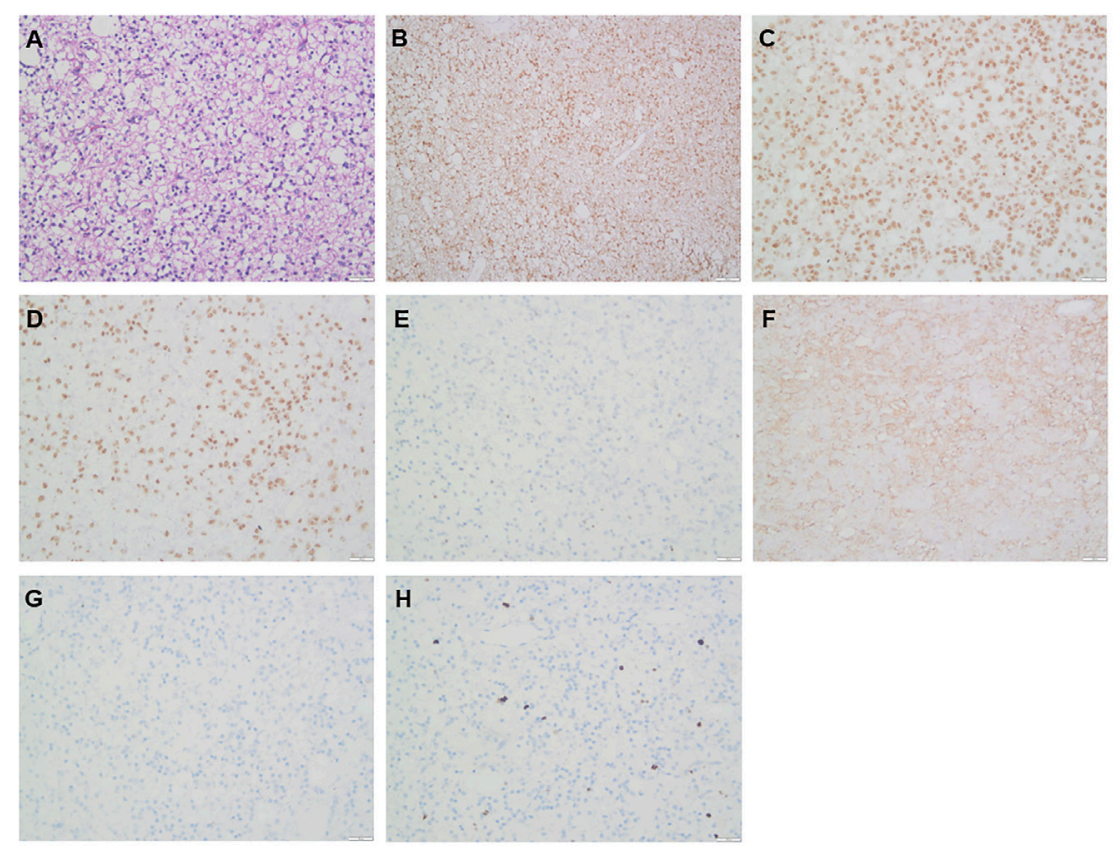

I

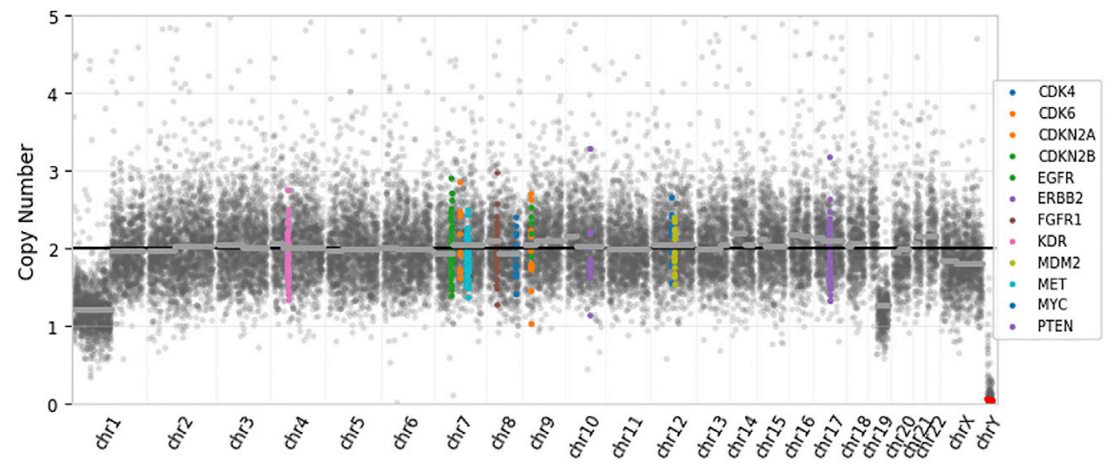

J
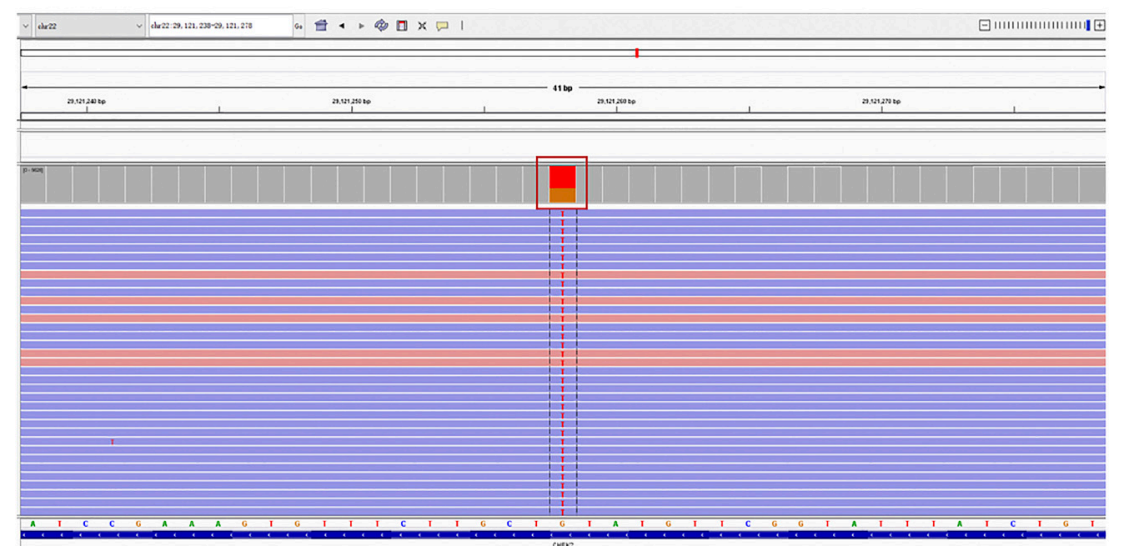

FIGURE 1 | Histopathologic stains from the postoperative right frontal lobe (A-H), 1P19Q chromosomal combination deletion (I) and the Integrative Genomics Viewer snapshot of CHEK2 p.R137* (J). (A) hematoxylin and eosin. (B) IDH. (C) ATRX. (D) Olig2. (E) P53. (F) GFAP. (G) NeuN. (H) Ki67. (200x). 


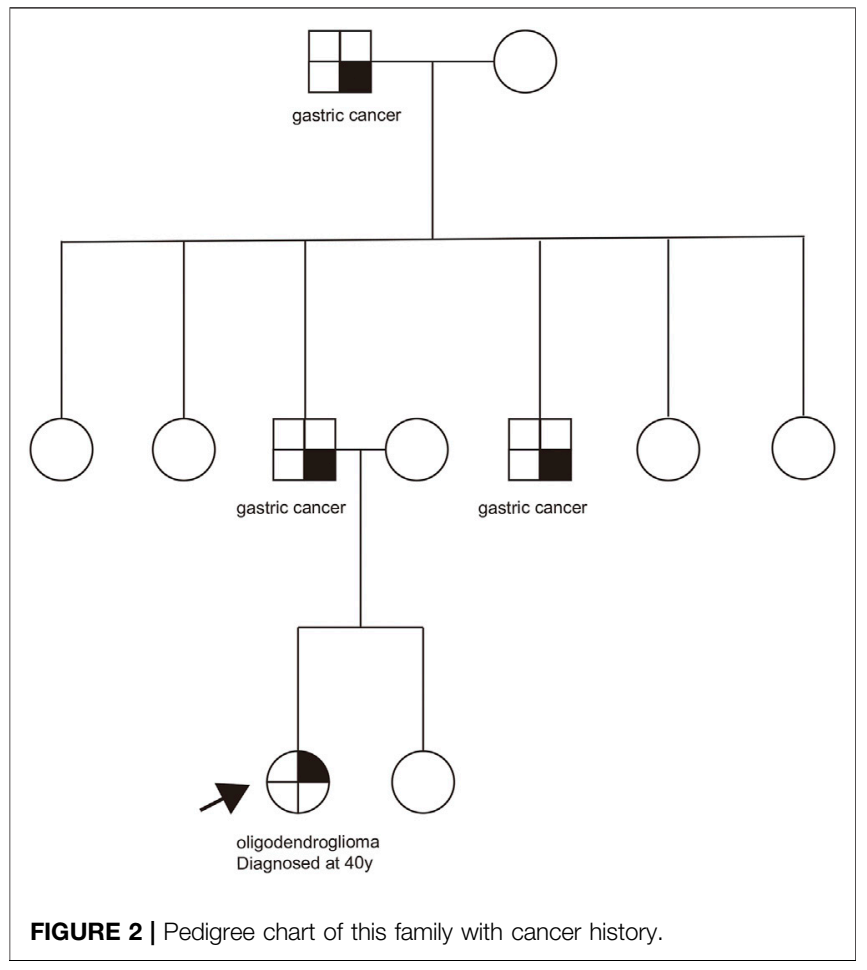

CHEK2 truncating and missense mutations might be different. The association between CHEK2 and other cancers, including the male breast, kidney, gastric, prostate, lung, ovarian, and thyroid cancers, was also reported (Cybulski et al., 2004). Germline CHEK2 mutations have also been observed in medulloblastoma and primary glioblastomas (Sallinen et al., 2005). Currently, there is no direct evidence supporting the relationship of CHEK2 with CNS tumors. Herein, we reported a p.R137* mutation that occurred in the CHEK2 FHA domain in a Chinese female patient with oligodendroglioma for the first time.

\section{CASE PRESENTATION}

A 40-year-old female was admitted to hospital on 30 June 2020 for sudden loss of consciousness for 17 days. Physical examination showed binocular vision decline. Brain magnetic resonance imaging (MRI) was performed, and it showed irregular and abnormal signal shadows in the right frontal lobe, low signal in $\mathrm{T} 1$ and high signal in $\mathrm{T} 2$, no obvious enhancement in lesions on enhanced scan, mild compression of the surrounding brain tissue, and no significant shift in the midline structure. Diagnosis on admission showed the right frontal lobe space occupying the lesion and possibly symptomatic epilepsy. After the exclusion of surgical contraindications, the right frontal lobe glioma was removed under general anesthesia on 3 July 2020 .
Postoperative pathology showed that the morphology of the right frontal lobe was consistent with oligodendroglioma (WHO Grade II), IDH (+), ATRX (+), Olig-2(+), P53 (-), GFAP (-), NeuN (-), and ki-67 (10\%) (Figure 1). Nextgeneration sequencing (NGS) $131+4$ panel (Simceredx) profiling was performed using the postoperative tissue, and IDH1 R132H (allele frequency, AF 35.1\%), TERT C228T (AF $38.02 \%)$, and 1P19Q chromosomal combination deletions (Figure 1I) were identified, which indicated that the tumor had typical molecular characteristics of oligodendroglioma. Moreover, CHEK2 p.R137* (AF 49.36\%) was also detected in the tumor tissue. Then, considering the family history of tumors, we confirmed the mutation as a germline heterozygous variation using leukocytes (Figure 1J). Somatic mutation of CHEK2 and the deleterious variant of the TP53 gene were absent.

The patient had no history of other tumors, and her grandfather, father, and uncle all suffered from gastric cancer in her family (Figure 2). The family history of the patient excluded Li-Fraumeni/Li-Fraumeni-like syndrome (Giacomazzi et al., 2015). Due to the death of the tumor patients in the family, further family co-segregation analysis was not conducted.

One month after surgery, considering that the patient was older than 40 years old and in accordance with the patient's wishes, three-dimensional conformal radiotherapy (54 Gy/ 27F) and concurrent temozolomide chemotherapy (120 mg/ qd) were administered. One month after concurrent chemoradiotherapy, six cycles of $240 \mathrm{mg} / \mathrm{qd}$ adjuvant temozolomide chemotherapy were given on days $1-5$ every 4 weeks. At the time of writing, the patient has been discharged from the hospital and returned to work. According to the National Comprehensive Cancer Network (NCCN) guidelines, a woman with a germline $C H E K 2$ pathogenic variant has an estimated 15-40\% overall risk of breast cancer, and an annual mammogram and consideration of tomosynthesis and breast MRI with contrast were recommended for the patient in our case.

\section{DISCUSSION}

CHEK2, a serine/threonine kinase gene, plays an essential role in the DNA damage checkpoint pathway, which can prevent damaged cells from entering mitosis or arrest the cell cycle (Apostolou and Papasotiriou, 2017). CHEK2 is also required for chromosomal stability during mitosis (Stolz et al., 2010). The p.R137* mutation occurred in the functionally important domain of CHEK2 (the FHA domain), resulting in the loss of the protein kinase domain and promoting tumor formation. CHEK2 p.R137* has been previously seen in very low population frequencies in TOPMed, gnom $A D$, and ExAC. This mutation is listed in the ClinVar database (https:// www.ncbi.nlm.nih.gov/clinvar/variation/182452/) and has only been observed in individuals affected with breast 
cancer (Leedom et al., 2016), and this case would be an informative addition.

Although it is not clear at present if the CHEK2 p.R137* variant is associated with the oligodendroglioma development in this patient, the heterozygous germline CHEK2 or TP53 pathogenic variants have been reported in patients with brain tumors (Bougeard et al., 2015; Valdez et al., 2017; Aedma and Kasi, 2020). In consideration of the unavailability of family patient samples, the relationship between CHEK2 mutations and CNS tumors needs more research and reports in the future. Our case is a patient with an oligodendroglioma phenotype harboring the CHEK2 p. R137* mutation, suggesting her siblings and offspring would have a $50 \%$ chance of having the same variant, which may increase the tumor risk.

The treatment options for oligodendroglioma are limited. Usually, primary surgery followed by postoperative radiotherapy and chemotherapy is performed (Weller et al., 2017). CHEK2 is an important gene in the DNA homologous recombination repair (HRR) pathway. Inactivation of the ATMCHEK2 pathway has been reported to sensitize human tumors to radio/chemotherapy based on cisplatin, temozolomide, and poly (ADP-ribose) polymerase inhibitors (Manic et al., 2015). Currently, the FDA has approved olaparib for metastatic castration-resistant prostate cancer patients with defective HRR genes. In the future, olaparib combined with temozolomide/radiotherapy might be a promising treatment option for patients with CNS tumors harboring the CHEK2 germline mutation.

In our case, there are some limitations. This family has no cancers typically associated with the CHEK2 pathogenic variant, and in this family, the patient's grandfather, father, and uncle all expired, so the pedigree verification was not conducted. There was no loss of heterozygosity ( $\mathrm{LOH}$ ) found in the tumor tissue in our case. Therefore, further studies are needed to confirm the association between the CHEK2 gene and oligodendroglioma.

\section{REFERENCES}

Aedma, S. K., and Kasi, A. (2020). Li-Fraumeni Syndrome. Treasure Island (FL): StatPearls Publishing. Available from: https://www.ncbi.nlm.nih.gov/books/ NBK532286/.

Apostolou, P., and Papasotiriou, I. (2017). Current Perspectives on CHEK2 Mutations in Breast Cancer. Breast Cancer (Dove Med. Press. Vol. 9, 331-335. doi:10.2147/BCTT.S111394

Bartek, J., and Lukas, J. (2003). Chk1 and Chk2 Kinases in Checkpoint Control and Cancer. Cancer Cell 3 (5), 421-429. doi:10.1016/s1535-6108(03)00110-7

Bougeard, G., Renaux-Petel, M., Flaman, J.-M., Charbonnier, C., Fermey, P., Belotti, M., et al. (2015). Revisiting Li-Fraumeni Syndrome from TP53 Mutation Carriers. Jco 33, 2345-2352. doi:10.1200/JCO.2014.59.5728

Cybulski, C., Górski, B., Huzarski, T., Masojć, B., Mierzejewski, M., Dębniak, T., et al. (2004). CHEK2 Is a Multiorgan Cancer Susceptibility Gene. Am. J. Hum. Genet. 75 (6), 1131-1135. doi:10.1086/426403

Giacomazzi, C. R., Giacomazzi, J., Netto, C. B. O., Santos-Silva, P., Selistre, S. G., Maia, A. L., et al. (2015). Pediatric Cancer and Li-Fraumeni/Li-fraumeni-like Syndromes: a Review for the Pediatrician. Rev. Assoc. Med. Bras. 61 (3), 282-289. doi:10.1590/1806-9282.61.03.282

\section{CONCLUSION}

To our knowledge, this is the first report that we identified a pathogenic variant CHEK2 p.R137* in an oligodendroglioma patient, which suggests that brain tumor patients with a family history of cancer or other risk factors need to pay more attention to the potential genetic predisposition variant for moderate/low penetrance genes. This may provide the direction for future treatments.

\section{DATA AVAILABILITY STATEMENT}

The original contributions presented in the study are included in the article/Supplementary Materials, further inquiries can be directed to the corresponding author.

\section{ETHICS STATEMENT}

Written informed consent was obtained from the [individual(s) AND/OR minor(s)' legal guardian/next of kin] for the publication of any potentially identifiable images or data included in this article.

\section{AUTHOR CONTRIBUTIONS}

DJ designed the study; XL drafted the manuscript; HX and TH analyzed the literature; NL revised the manuscript; ML participated in the revision. All authors contributed to the article and approved the submitted version.

\section{ACKNOWLEDGMENTS}

The authors thank Chuang Qi, Xueyu Yang, Wanglong Deng, Guanghua $\mathrm{Lu}$, and Ran Ding from Simceredx for their kind assistance.

Jalilvand, M., Oloomi, M., Najafipour, R., Alizadeh, S. A., Saki, N., Rad, F. S., et al. (2017). An Association Study between CHEK2 Gene Mutations and Susceptibility to Breast Cancer. Comp. Clin. Pathol. 26 (4), 837-845. doi:10.1007/s00580-017-2455-x

Leedom, T. P., LaDuca, H., McFarland, R., Li, S., Dolinsky, J. S., and Chao, E. C. (2016) Breast Cancer Risk Is Similar for CHEK2 Founder and Non-founder Mutation Carriers. Cancer Genet. 209 (9), 403-407. doi:10.1016/j.cancergen.2016.08.005

Manic, G., Obrist, F., Sistigu, A., and Vitale, I. (2015). Trial Watch: Targeting ATM-CHK2 and ATR-CHK1 Pathways for Anticancer Therapy. Mol. Cell Oncol. 2 (4), e1012976. doi:10.1080/23723556.2015.1012976

Sallinen, S.-L., konen, T., Haapasalo, H., and Schleutker, J. (2005). CHEK2 Mutations in Primary Glioblastomas. J. Neurooncol. 74 (1), 93-95. doi:10. 1007/s11060-005-5953-7

Stolarova, L., Kleiblova, P., Janatova, M., Soukupova, J., Zemankova, P., Macurek, L., et al. (2020). CHEK2 Germline Variants in Cancer Predisposition: Stalemate rather Than Checkmate. Cells 9 (12), 2675. doi: $10.3390 /$ cells9122675

Stolz, A., Ertych, N., Kienitz, A., Vogel, C., Schneider, V., Fritz, B., et al. (2010). The CHK2-BRCA1 Tumour Suppressor Pathway Ensures Chromosomal Stability in Human Somatic Cells. Nat. Cel Biol 12 (5), 492-499. doi:10.1038/ncb2051

Valdez, J. M., Nichols, K. E., and Kesserwan, C. (2017). Li-Fraumeni Syndrome: a Paradigm for the Understanding of Hereditary Cancer 
Predisposition. Br. J. Haematol. 176, 539-552. doi:10.1111/bjh. 14461

Weller, M., van den Bent, M., Tonn, J. C., Stupp, R., Preusser, M., Cohen-Jonathan-Moyal, E., et al. (2017). European Association for Neuro-Oncology (EANO) Guideline on the Diagnosis and Treatment of Adult Astrocytic and Oligodendroglial Gliomas. Lancet Oncol. 18 (6), e315-e329. doi:10.1016/S1470-2045(17)30194-8

Conflict of Interest: Authors NL, TH, and ML were employed by Jiangsu Simcere Diagnostics Co., Ltd.

The remaining authors declare that the research was conducted in the absence of any commercial or financial relationships that could be construed as a potential conflict of interest.
Publisher's Note: All claims expressed in this article are solely those of the authors and do not necessarily represent those of their affiliated organizations, or those of the publisher, the editors, and the reviewers. Any product that may be evaluated in this article, or claim that may be made by its manufacturer, is not guaranteed or endorsed by the publisher.

Copyright $\odot 2022 \mathrm{Li}$, Xue, Luo, Han, Li and Jia. This is an open-access article distributed under the terms of the Creative Commons Attribution License (CC BY).

The use, distribution or reproduction in other forums is permitted, provided the original author(s) and the copyright owner(s) are credited and that the original publication in this journal is cited, in accordance with accepted academic practice. No use, distribution or reproduction is permitted which does not comply with these terms. 